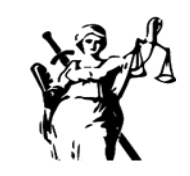

JUSTICIA

ISSN impreso 0124-7441
ISSN digital 2590-4566

\title{
Aspectos normativos de las finanzas en las universidades públicas del Caribe Colombiano
}

\section{Aspects normatives of finance in the public universities of the Colombian Caribbean}

\author{
(iD) \\ Andrés Villanueva Imitola \\ Universidad del Atlántico, Colombia \\ andresvillanueva@mail.uniatlantico.edu.co
}

\author{
(iD) \\ Elcira Solano Benavides \\ Universidad del Atlántico, Colombia \\ elcirasolano@mail.uniatlantico.edu.co
}

Recibido: 07 de noviembre de 2019 / Aceptado: 14 de febrero de 2020 https://doi.org/10.17081/just.25.38.4538

\section{Resumen}

El objetivo de este artículo. es describir la normatividad y la situación presupuestal relacionada con las finanzas de las universidades públicas del Caribe Colombiano que forman parte del Sistema Universitario Estatal del Caribe Colombiano - SUE Caribe. El método utilizado fue un estudio con un enfoque cuantitativo, dado que permitió indagar sobre la normatividad que rige las universidades estatales desde el punto de vista presupuestal, del mismo modo se analizaron las transferencias percibidas por las Universidades públicas del Caribe Colombiano. El tipo de investigación es de naturaleza descriptiva y explicativa. En los resultados se observó que las tasas de crecimientos de los aportes del Estado a las universidades han disminuido en términos de aportes por estudiantes. Mientras que los rubros financieros son significativos como es el caso de los recursos propios y los recursos del impuesto sobre la renta para la equidad (CREE). Dentro de las conclusiones se evidencia la importancia de los aspectos normativos para comprender la aplicación de los recursos financieros en las universidades públicas.

Palabras clave: normatividad, finanzas, educación superior pública, presupuesto.

\begin{abstract}
The objective de this article is to describe the regulations and budgetary situation related to the finances of the public universities of the Colombian Caribbean that are part of the State University System of the Colombian Caribbean - SUE Caribbean. The method used was a study with a quantitative approach, since it made it possible to investigate the regulations governing state universities from the budgetary point of view, Likewise, the transfers received by the public universities of the Colombian Caribbean were analyzed. The type of research is descriptive and explanatory in nature. The results showed that the growth rates of State contributions to universities have decreased in terms of student contributions. While the financial items are significant as is the case of own resources and income tax resources for equity (CREE). The conclusions highlight the importance of regulatory aspects to understand the application of financial resources in public universities.
\end{abstract}

Keywords: normatives, finance, public higher education, budget.

\section{Como citar:}

Villanueva Imitola, A., \& Solano Benavides, E. (2020). Aspectos normativos de las finanzas en las universidades públicas del Caribe Colombiano. Justicia, 25(38), 179-188. https://doi.org/10.17081/just.25.38.4538 


\section{INTRODUCCION}

La Constitución Política de Colombia en su artículo 69 establece la autonomía universitaria, a partir de la cual las universidades podrán proveer sus directivas y regirse por sus propios estatutos, de acuerdo con la ley, en ese sentido, se promulgó la ley 30 de 1992, "por la cual se organiza el servicio público de la educación superior", donde se creó un régimen especial para las universidades del Estado. De igual manera la norma establece que el Estado fortalecerá la investigación científica en las universidades oficiales y privadas y ofrecerá las condiciones especiales para su desarrollo, facilitando los mecanismos financieros que hagan posible el acceso de todas las personas a la educación superior.

Asimismo, la ley 30 de 1992, desde el punto de vista presupuestal determina que las fuentes de recursos de las universidades estatales son: los recursos provenientes de la nación, los recursos aportados por las entidades territoriales y la financiación aportada por los recursos propios de cada institución universitaria, los cuales se obtienen a través de venta de servicios, matrículas, derechos académicos, programas de extensión, consultorías o convenios de investigación, entre otros. El presente artículo se propone describir la normatividad desde el punto de vista presupuestal y el comportamiento de las transferencias de la nación a las Universidades que conforman el Sistema Universitario Estatal del Caribe Colombiano - SUE Caribe en el periodo 2009 -2017. Es de anotar, que el SUE Caribe es la Red de Universidades Estatales en el Caribe Colombiano, su declaratoria misional la promulga la Ley 30 de 1992, donde se legitima acuerdos y convenios de investigación y cooperación desde 1997 y la asumen con responsabilidad y confiabilidad los Rectores, Directivos Académicos de las Universidades que conforman la Red.

En cuanto, a los recursos financieros de las Universidades se encuentran enmarcados en el artículo 69 de la Constitución Política Colombia y provistos por la nación y el ente territorial (en el caso que sea financiada por las gobernaciones), estos recursos son asignados por ley de acuerdo a lo dispuesto en los artículos 86 y 87 del capítulo 5, título tercero de la ley 30 de 1992. Al abordar el financiamiento de las universidades públicas se hace necesario definir la conceptualización de finanzas públicas, por consiguiente, se puede definir desde tres puntos de vista diferentes: El primero, se define como los recursos disponibles por parte del Estado y las entidades públicas para el cumplimiento de sus actividades y proyectos. El segundo, como el conjunto de entidades públicas que tienen encomendado gestionar los ingresos que recibe el Estado. El tercero, que corresponde al más común de todos, como la disciplina que se encarga del estudio de los objetivos del sector público y la forma como se pueden lograr estos con unos recursos limitados (Economía Planeta, 1980).

\section{Método}

El estudio se fundamenta en un enfoque cuantitativo, dado a que permitió indagar sobre la normatividad que rige las universidades estatales desde el punto de vista presupuestal, del mismo modo se analizaron las transferencias percibidas por las Universidades públicas del Caribe Colombiano. El tipo de investigación es de naturaleza descriptiva y explicativa. Es descriptiva, dado que su finalidad es la descripción de la normatividad que rige la educación superior desde el punto de vista presupuestal y es explicativa porque propone explicar y analizar el comportamiento de las transferencias por parte de la nación a las universidades objeto de estudio. 


\section{Resultados}

\section{Aspectos normativos de las Universidades Públicas}

Las Universidades públicas, tiene un régimen especial en su calidad de Universidad del Estado; por lo tanto, el Título III de la Ley 30 de 1992 establece las condiciones para su funcionamiento. Asimismo, en el inciso 3o del artículo 57 de la mencionada ley se les reconoce autonomía “académica, administrativa y financiera, patrimonio independiente y podrán elaborar y manejar su presupuesto de acuerdo con las funciones que le corresponden". El carácter especial del régimen de las universidades estatales u oficiales comprenderá el régimen financiero y el régimen de contratación y control fiscal, de acuerdo con la ley 30 de 1992.

De igual manera, en el artículo tercero de la Ley 30 de 1992, establece la autonomía universitaria y vela por la calidad del servicio educativo a través del ejercicio de la suprema inspección y vigilancia de la Educación Superior. Entre tanto en el artículo 28 desarrolló los aspectos en que se refleja la autonomía universitaria, de la siguiente manera:

“Artículo 28. La autonomía universitaria consagrada en la Constitución Política de Colombia y de conformidad con la presente ley, reconoce a las universidades el derecho de darse y modificar sus estatutos, designar sus autoridades académicas y administrativas, crear, organizar y desarrollar sus programas académicos, definir y organizar sus labores formativas, académicas, docentes, científicas y culturales, otorgar los títulos correspondientes, seleccionar a sus profesores, admitir a sus alumnos y adoptar sus correspondientes regímenes y establecer, arbitrar y aplicar sus recursos para el cumplimiento de su misión social y su función institucional".

Adicionalmente, la Ley 30 de 1992 estableció que, el Gobierno Nacional incrementará sus aportes para las universidades estatales u oficiales en un porcentaje no inferior al 30\% del incremento del Producto Interno Bruto. Para tal efecto, la distribución de esos recursos, el Ministerio de Educación Nacional en conjunto con el Sistema Universitario Estatal del Caribe Colombiano, elaboró una propuesta de distribución basada en indicadores de desempeño de las instituciones de educación superior atendiendo a criterios de eficiencia y desempeño de las universidades, con el fin de hacer seguimiento a los recursos y el cumplimiento de las metas trazadas.

Desde la perspectiva financiera de las Universidades Públicas, el presupuesto es el instrumento financiero de corto plazo, utilizado para arbitrar los recursos de que dispone una entidad, para el cumplimiento de su función, el cual se debe se promulga a través de un acto administrativo por el cual se computan anticipadamente las rentas e ingresos de la entidad que esperan recibir en una determinada vigencia fiscal. Lo mismo que los gastos y apropiaciones en que incurran todos los órganos que lo integran y refleja las proyecciones del plan financiero.

De igual manera, en materia financiera el presupuesto es el instrumento operativo básico y de su ejecución anual depende el cumplimiento de las metas fijadas por la administración. Por lo tanto, el proceso de preparación debe ser abordado técnicamente para evitar la sobreestimación de ingresos, la subvaloración de gastos o viceversa.

Por lo tanto, existen diversas opiniones acerca de lo que significa el presupuesto público, (Ramírez, 1998) considera que es el cálculo prospectivo de ingresos y gastos públicos para un período concreto, dirigido al sostenimiento de los servicios públicos y regulación económica y dentro del plan económico de la Nación. En este contexto de las finanzas, es necesario anotar, el concepto de administración financiera, la cual estudia las decisiones administrativas que conducen a la adquisición y financiamiento de los activos, 
manejo de los pasivos, problemas de tamaño y crecimiento de las organizaciones (Philippatos, 1974), otros autores definen la gerencia financiera como la asignación de fondos dentro de la empresa y la adquisición de los mismos (Van Horne, 1998). De igual manera, se concibe la gerencia financiera como la ciencia y el arte de administrar el dinero (Gitman, 2000).

Por otra parte, la gestión financiera es un proceso que involucra los ingresos y egresos atribuibles a la realización del manejo racional del dinero en las organizaciones y en consecuencia, la rentabilidad financiera generada por el mismo. Por ende, nos permite definir el objetivo básico de la gestión financiera desde dos elementos: la generación de recursos o ingresos, incluyendo los aportes de los asociados y en segundo lugar, la eficiencia y eficacia o esfuerzos y exigencias en el control de los recursos financieros, para obtener niveles aceptables y satisfactorios en su manejo (Sánchez, 2006).

Analizando un poco más el tercer punto de vista, vemos que, para lograr sus objetivos, las entidades públicas deben realizar tres actividades fundamentales: 1. Obtención de ingresos y otros recursos, 2. Ejecución y control de los gastos e inversiones y 3. Elaboración y posterior control del presupuesto necesario para realizar las actividades propuestas. (Villanueva, Florentino y Rico, 2015). En ese mismo sentido el objeto de las finanzas públicas es la redistribución para dar un mayor bienestar a la población por medio de una distribución más equitativa de los recursos, la estabilidad y por último el desarrollo de la economía (Restrepo, 2000).

\section{Estructura del Presupuesto de las Universidades estatales}

Desde el punto de vista de la estructura del presupuesto de las universidades públicas se encuentran regulada por lo establecido en el Decreto 111 de 1996, donde señala la siguiente estructura:

SISTEMA PRESUPUESTAL. El sistema presupuestal está constituido por el plan financiero, el plan operativo anual de inversiones y por el Presupuesto general de la institución, atendiendo el Decreto 111 de 1996. El Plan Financiero, es el instrumento de planificación y gestión financiera que tiene como base las operaciones efectivas de la institución, tomando en consideración las previsiones de ingresos, gastos, déficit y su financiación, compatibles con el Plan Anual Mensualizado de Caja - PAC. El Plan Operativo anual de Inversiones, conformado por los proyectos de inversión, clasificados por origen de los recursos, por unidad ejecutora y programas, y el Presupuesto general. el cual se encuentra compuesto por: el Presupuesto de Rentas, el presupuesto de gastos o apropiaciones y las disposiciones generales. El presupuesto de rentas, contendrá la estimación de los ingresos corrientes (transferencias de entidades territoriales y los recursos propios) y de los recursos de capital. El Presupuesto de gastos o apropiaciones, incluye los gastos de funcionamiento (servicios personales, gastos generales y transferencias), servicio de la deuda y gastos de inversión, y por último las disposiciones generales: Hace referencia a las normas de cada uno de los rubros, tendientes a asegurar la correcta ejecución del presupuesto anual.

De acuerdo con lo establecido en el plan financiero, las instituciones públicas, deben tener un plan anual mensualizado de caja (PAC), el cual constituye el instrumento mediante el cual se define el monto máximo mensual de fondos disponibles, y el monto máximo mensual de pagos. En consecuencia, los pagos se harán teniendo en cuenta el PAC y se sujetarán a los montos aprobados en él. Por lo tanto, el PAC corresponde a las apropiaciones de cada vigencia fiscal y tendrá como límite máximo el valor del presupuesto de ese periodo. (Urrego \& Gutierrez, 2018). 


\section{Principios del Sistema Presupuestal.}

Dentro de los principios presupuestales consagrados en el Estatuto orgánico del presupuesto o Decreto 111 de 1996, se encuentran los siguientes:

$\gg$ 1. Planificación. El Presupuesto General de la Universidad deberá guardar concordancia con los contenidos del Plan de Desarrollo institucional, del plan financiero y del plan operativo anual de inversiones.

$\gg$ 2. Anualidad. El año fiscal comienza el 1‥ De enero y termina el 31 de diciembre de cada año. Después del 31 de diciembre, no podrán asumirse compromisos con cargo a las apropiaciones del año fiscal que se cierra en esa fecha y los saldos de apropiación no afectados por compromisos, caducarán sin excepción.

$\gg$ 3. Universalidad. Los estimativos de ingresos incluirán el total de los provenientes de aportes, rentas propias y recursos de capital, que se esperen recibir o reciban durante el año fiscal, sin deducción alguna. El presupuesto de gastos, contendrá la totalidad de los gastos que se espere realizar durante la vigencia fiscal respectiva. En consecuencia, ninguna autoridad podrá efectuar gastos, erogaciones con cargo al tesoro o transferir crédito alguno, que no figure en el presupuesto.

$\gg$ 4. Unidad de Caja. Con el recaudo de todos los aportes, rentas y recursos de capital, se atenderá la situación de fondos de las unidades ejecutoras, para el pago oportuno de las apropiaciones autorizadas en el Presupuesto General de la Universidad.

$\gg$ 5. Programación Integral. Todo programa presupuestal deberá contemplar simultáneamente los gastos de inversión y de funcionamiento que las exigencias técnicas y administrativas demanden como necesarios para su ejecución y operación, de conformidad con los procedimientos y normas legales vigentes.

$\gg$ 6. Las apropiaciones deben referirse en cada programa presupuestal a su objeto y funciones, y se ejecutarán estrictamente conforme al fin para el cual fueron programadas.

$\gg$ 7. Inembargabilidad. Son inembargables las rentas incorporadas en el Presupuesto General de la Universidad del Atlántico, asi como los bienes y derechos de las Unidades Académico Administrativas que la conforman.

$\gg$ 8. Coherencia Macroeconómica. El Presupuesto de la Universidad del Atlántico debe ser compatible con las metas macroeconómicas fijadas por el Gobierno Nacional en coordinación con la Junta Directiva del Banco de la República.

$\gg$ 9. Homeostasis Presupuestal. El crecimiento real del presupuesto de rentas de la Universidad del Atlántico incluida la totalidad de los créditos adicionales de cualquier naturaleza, deberá guardar congruencia con el crecimiento de la economía, de tal manera que no genere desequilibrio macroeconómico.

Desde el punto de vista cuantitativo, los aportes de la Nación en valores corrientes a las universidades SUE Caribe en el periodo 2009-2017 crecieron en promedio 6,43\%. Para el 2009 estos recursos fueron de $\$ 285.347$ millones de pesos, mientras que para el 2017 fue de 467.649 millones de pesos. A partir del año 2013, se observa una disminución en el crecimiento de las transferencias con respecto al 2012, luego presenta una leve recuperación en el 2016 hasta llegar al año 2017 con un incremento equivalente del 9\%. Ver Grafica 1. 
Grafica 1. Aportes de la Nación en transferencias a las universidades SUE Caribe 2009-2017.

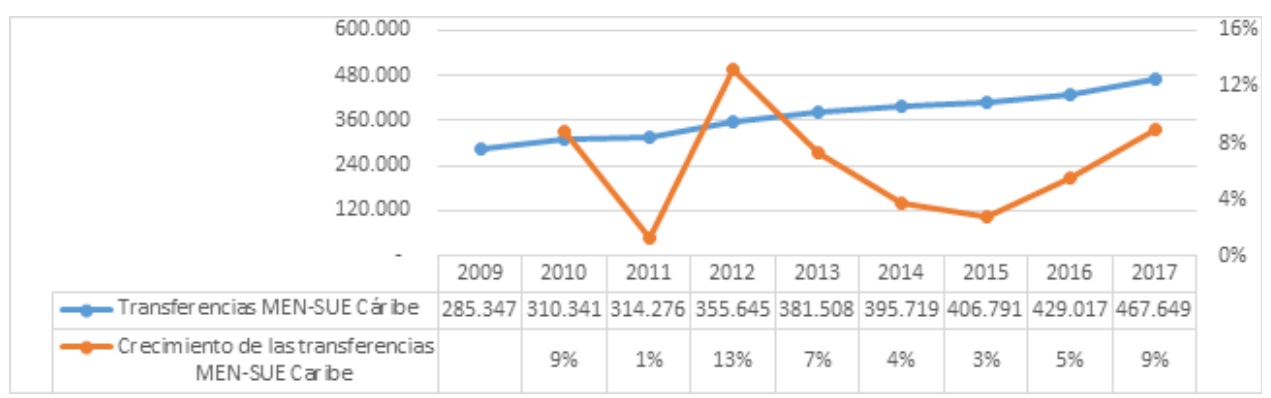

Fuente: Elaboración propia, con base a datos del Ministerio de Educación Nacional. No se incluyen los recursos del CREE y del CESU

En cuanto al análisis de los aportes financieros en valores corrientes para la educación pública por estudiante matriculados en la región Caribe, se muestra como estos aportes disminuyen por parte de la Nación. En el año 2010, se presenta el mayor aporte por estudiantes del periodo con $\$ 4.265$ millones de pesos. Después de este año, el aporte por estudiantes disminuyó a 3.581 millones de pesos, sin embargo, las brechas en el aporte por estudiantes en el 2011 fueron de $\$ 684$ millones con respecto al 2010, \$471 millones en el 2012, $\$ 533$ millones en el 2013, $\$ 429$ millones en el 2014, $\$ 464$ millones en el 2015, $\$ 317$ millones en el 2016 y $\$ 81$ millones de pesos en el 2017, es decir, cada año la brecha disminuye pero aún no se logra superar el aporte del 2010. Con respecto a la tasa de crecimiento de aportes por estudiantes se observa que en los años 2011, 2013 y 2015 tienen tasas de crecimiento negativas. Ver. Fuente: Elaboración propia, con base a

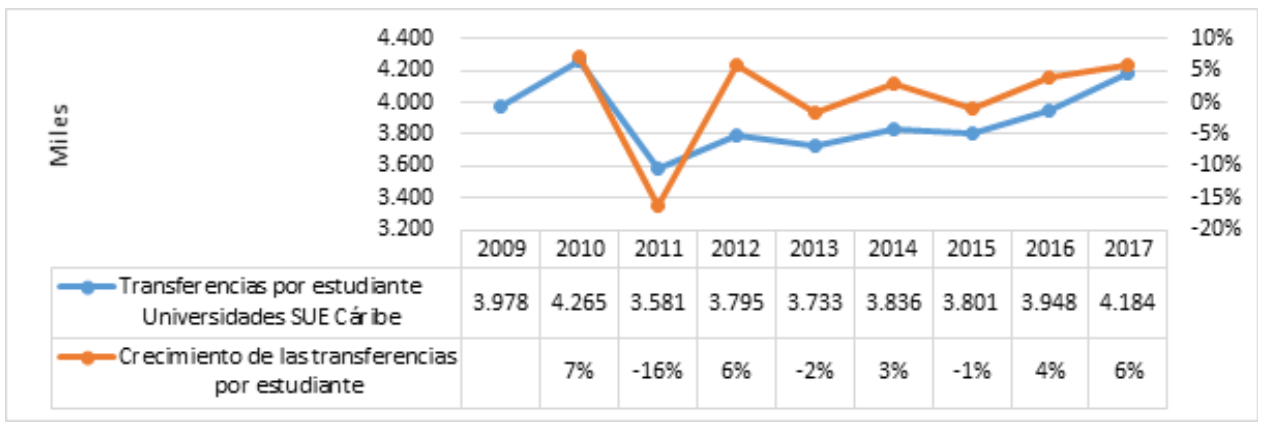

datos del Ministerio de Educación Nacional. No se incluyen los recursos del CREE y del CESU..

Grafica 2. Transferencias por estudiante universidades SUE Caribe 2009-2017.

Fuente: Elaboración propia, con base a datos del Ministerio de Educación Nacional. No se incluyen los recursos del CREE y del CESU.

Según el artículo 86 de la Ley 30 de 1992, los aportes de la Nación a las universidades oficiales deben ser a pesos constante, es decir, deben tener en cuenta la inflación para que las universidades no pierdan poder adquisitivo. Cuando se relacionan los aportes de la Nación a las universidades SUE Caribe en el periodo 2009-2017 con el IPC, se evidencia una pérdida en poder adquisitivo de estos aportes en tres años, es decir, el IPC está por encima del crecimiento de los aportes y los recursos dejados de recibir por las universidades fueron de (7.738) millones en 2011, (16.158) millones en 2015 y (1.227) millones en 2016. En los años 2010, 2012, 
2013, 2014 y 2017, el crecimiento de los aportes está por encima del IPC, ganando las universidades poder adquisitivo.

Cabe anotar, que, para la financiación de la educación superior pública, el gobierno y estudiantes han logrado acuerdos donde establece un aumento de los aportes a la base al índice de precios al consumidor (IPC) + 3,5\% en 2019, IPC + 4\% en 2020, IPC + 4,5\% en 2021 e IPC + 4,65\% en 2022.

Además, se observa un comportamiento contra-cíclico entre las dos variables, excepto en el 2013, es decir, mientras las transferencias crecen, el IPC cae. Por tal razón, se infiere que el Estado realiza la causación de los ajustes por IPC en el periodo siguiente, produciendo una pérdida en el poder adquisitivo

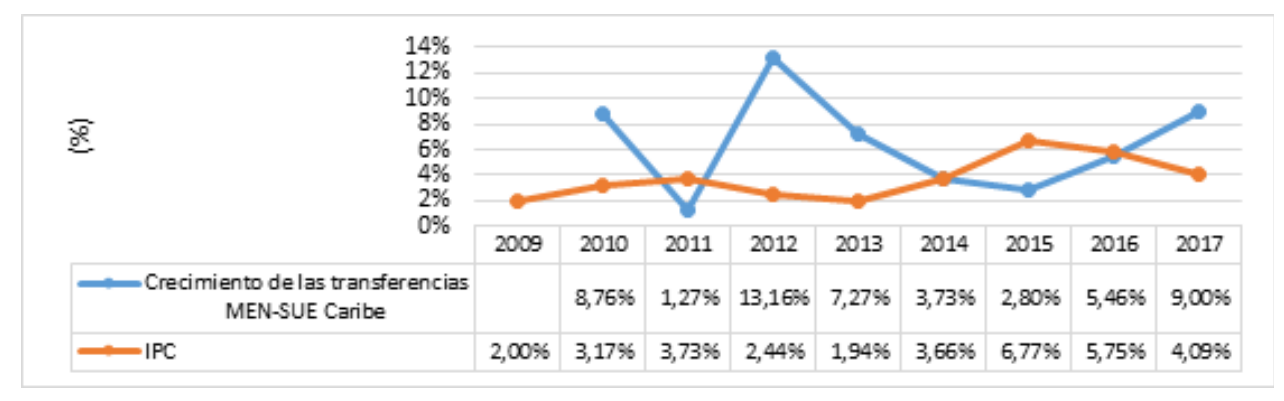

en las universidades. Ver Grafica 3.

Grafica 3. Crecimiento de las transferencias de la Nación a las universidades SUE Caribe y variación del IPC de Colombia 2009-2017.

Fuente: Elaboración propia, con base a datos del Ministerio de Educación Nacional. No se incluyen los recursos del CREE y del CESU

Según el artículo 87 de la Ley 30 de 1992, las universidades oficiales deben incrementar sus aportes en un porcentaje no inferior al $30 \%$ del incremento real del PIB. Sin embargo, la distribución de estos recursos quedó condicionado a los artículos 81 y 82 de la misma Ley, en donde el Consejo Nacional de Educación Superior determina esta distribución sujeta a la racionalidad, evaluación y optimización de los recursos. Desde el año 2003 se creó un modelo de distribución de estos recursos, de tal manera que las universidades públicas competían por estos recursos con sus indicadores de Capacidad (metros2, números de docentes tiempo completo, Recursos financieros y gastos en personal administrativos), Índice de Formación (número de matriculados, graduados, número de programas, graduados vinculados al mercado formal, estudiantes con alto nivel de inglés y movilidad), Índice de investigación, Índice Extensión e Îndice de bienestar.

Según el artículo 87 de la Ley 30, el crecimiento de los aportes debería ser procíclicos con el crecimiento del PIB. Cuando se comparan estos dos ingresos, se evidencia que son contra cíclicos en todos los años, excepto en 2014 y 2015; es decir, el crecimiento del PIB se mueve en dirección opuesta a las transferencias. Por otro lado, en el 2014, el crecimiento del PIB pasa de 4,9\% al 4,4\%y el crecimiento de los aportes disminuye del 7,3\% al 3.7\%. Similarmente, en el 2015, el crecimiento del PIB pasó de 4,4\% al 3,1\% y el crecimiento de los aportes pasa en $3,7 \%$ a $2,8 \%$. Ver Grafica 4 y 5. 
Grafica 4. Crecimiento de las transferencias de la Nación a las universidades SUE Caribe y crecimiento del PIB de Colombia 2009-2017.

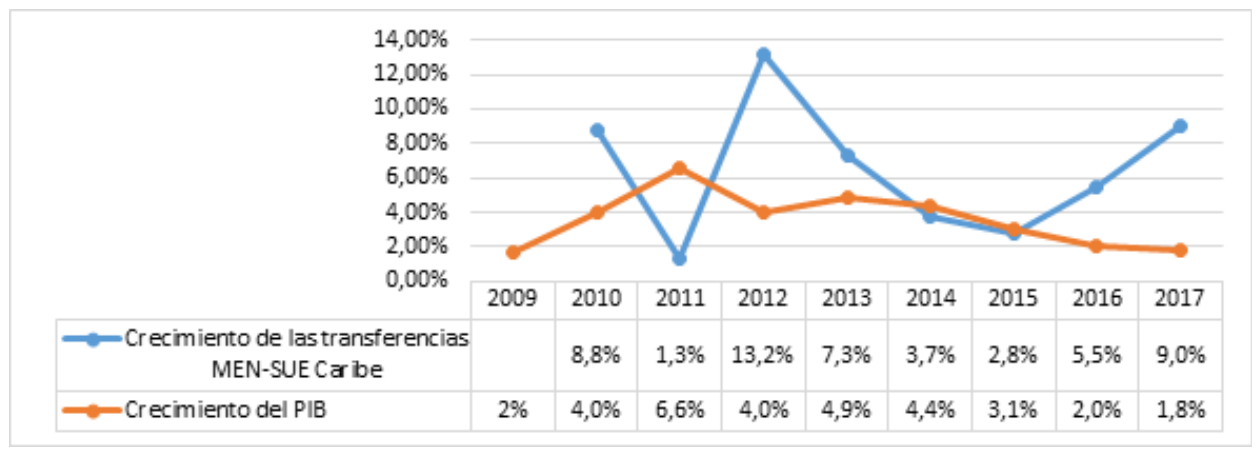

Fuente: Elaboración propia, con base a datos del Ministerio de Educación Nacional. No se incluyen los recursos del CREE y del CESU

Grafica 5. Crecimiento de las transferencias por estudiante universidades SUE Caribe y crecimiento del PIB 2009-2017.

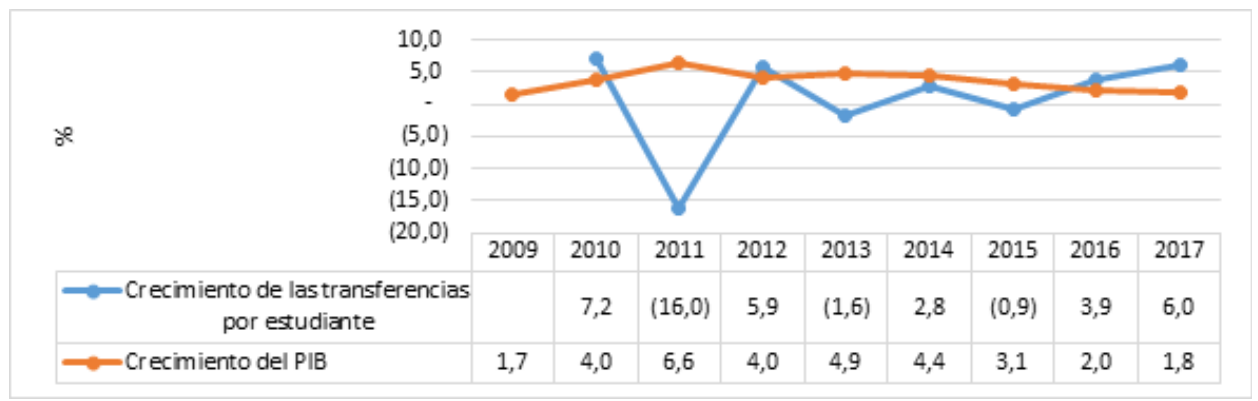

Fuente: Elaboración propia, con base a datos del Ministerio de Educación Nacional. No se incluyen los recursos del CREE y del CESU

Desde el punto de vista de los ingresos se evidencia una marcada dependencia de las transferencias de la nación por parte de las universidades, que por ley debe transferirles a las instituciones de educación superior públicas, además existe una baja participación de los recursos propios en el presupuesto total. Cabe anotar, la normatividad existente desde el punto de vista presupuestal, donde establece en el artículo 12, del Decreto 111 de 1996, los principios del sistema presupuestal los siguientes: la planificación, la anualidad, la universalidad, la unidad de caja, la programación integral, la especialización, inembargabilidad, la coherencia macroeconómica y la homeostasis.

Asimismo, se muestra los graduados por nivel de formación y los matriculados por nivel académico para el periodo 2018, de las Universidades SUE Caribe, las cuales se detallan en la tabla 1 y 2: 
TABLA 1. GRADUADOS POR NIVEL DE FORMACIÓN 2018 UNIVERSIDADES SUE CARIBE

\begin{tabular}{|l|l|l|l|l|l|l|l|l|}
\hline \multirow{2}{*}{$\begin{array}{c}\text { Nivel } \\
\text { de Formación }\end{array}$} & \multicolumn{7}{|c|}{ Universidades } \\
\cline { 2 - 10 } & Córdoba & Sucre & Magdalena & $\begin{array}{r}\text { Popular } \\
\text { Cesar }\end{array}$ & Cartagena & Guajira & Atlántico & Total \\
\hline $\begin{array}{l}\text { Técnica } \\
\text { Profesional }\end{array}$ & 5 & 0 & 229 & 1 & 153 & 72 & 85 & 545 \\
\hline Tecnológica & 27 & 13 & 389 & 9 & 164 & 29 & 51 & 682 \\
\hline Universitaria & 1354 & 413 & 2775 & 1588 & 2704 & 1123 & 2732 & 12689 \\
\hline Especialización & 109 & 12 & 281 & 774 & 382 & 28 & 173 & 1759 \\
\hline Maestría & 42 & 2 & 90 & 0 & 241 & 22 & 41 & 438 \\
\hline Doctorado & 0 & 0 & 14 & 0 & 13 & 0 & 0 & 27 \\
\hline Total general & 1537 & 440 & 3778 & 2372 & 3657 & 1274 & 3082 & 16140 \\
\hline
\end{tabular}

De acuerdo a la tabla anterior, se observa que las Universidades del Atlántico y del Magdalena aportan cada una con el $22 \%$ del total de graduados por nivel de formación de las Universidades analizadas del SUE Caribe en periodo 2018, mientras que la Universidad Popular del Cesar aporta el 13\%.

Desde el punto de vista de los matriculados por nivel académico se observa que el $15 \%$ de los matriculados en el periodo 2018 corresponden a la Universidad de Cartagena del total de las Universidades analizadas del SUE Caribe, mientras que las universidades de Córdoba y Sucre aportan el $14 \%$ y el $0.04 \%$ cada una. Ver tabla 2

\section{TABLA 2. MATRICULADOS POR NIVEL ACADÉMICO 2018 UNIVERSIDADES SUE CARIBE}

\begin{tabular}{|c|c|c|c|c|c|c|c|c|}
\hline \multirow{2}{*}{$\begin{array}{c}\text { Nivel } \\
\text { Académico }\end{array}$} & \multicolumn{8}{|c|}{ Universidades } \\
\hline & Córdoba & Sucre & Magdalena & Cesar & Cartagena & Guajira & Atlántico & Total \\
\hline $\begin{array}{l}\text { Matrícula } \\
\text { Pregrado }\end{array}$ & 16480 & 5509 & 18312 & 16240 & 16554 & 14391 & 23657 & 111143 \\
\hline $\begin{array}{l}\text { Matrícula } \\
\text { Posgrado }\end{array}$ & 451 & 68 & 376 & 2530 & 1197 & 495 & 542 & 5659 \\
\hline $\begin{array}{l}\text { Matrícula } \\
\text { Total }\end{array}$ & 16931 & 5577 & 18688 & 18770 & 17751 & 14886 & 24199 & 116802 \\
\hline
\end{tabular}

Fuente: elaboración propia, 2020 con base al SNIES

\section{Discusiones}

Con base a los resultados del proceso de revisión normativa, teórica y recolección de información cuantitativa se destaca, que las universidades estatales cuentan con una normatividad desde el punto de vista financiero, las cuales se encentran definidas desde la constitución política de Colombia, la ley 30 de 1992 y el Decreto 111 de 1996, aunque éste universidades cuentan con su estatuto presupuestal que rigen el manejo presupuestal.

Los aportes de la Nación a las universidades pertenecientes al SUE Caribe en el período 2009-2014 crecieron en promedio $6.43 \%$, mientras que en el período $2003-2017$, se duplicaron el número de estudiantes matriculados en programas de pregrado $(139,5 \%)$ 
Desde el punto de vista de la distribución de los recursos al interior de las universidades se refleja que para el año 2014, las universidades tienen como mayor fuente de financiación los recursos provenientes de la nación. Con respecto a la tasa de crecimiento de aportes por estudiantes se observa que, en los años 2011, 2013 y 2015 tienen tasas de crecimiento negativas.

La educación superior en Colombia, es reglamentada por la Ley 30 de 1992 que define el carácter y autonomía de las Instituciones de Educación Superior -IES-, de igual manera establece el objeto de los programas académicos y los procedimientos de fomento, inspección y vigilancia. De igual manera, la Ley 30 de 1992, establece los aspectos relacionados con la financiación de la Universidad pública en Colombia, indica que los presupuestos de las universidades estarán constituidos por aportes del Presupuesto nacional destinados a funcionamiento e inversión, y de los recursos provenientes de los entes territoriales y de las rentas propias de cada institución.

\section{Financiación}

Este artículo es resultado de un proyecto financiado por convocatoria interna por parte de la Universidad del Atlántico. En la elaboración del artículo ambos autores participamos en la construcción de cada uno de los ítems del producto.

\section{Referencia Bibliográfica}

Asamblea Nacional Constituyente (1991). Constitución Política de Colombia

Banco Mundial (2018). Indicadores económicos. Disponible en: https://datos.bancomundial.org

Congreso de Colombia (1992). Ley № 30. Por el cual se organiza el servicio público de la Educación Superior. Bogotá, Colombia. Disponible en: https://www.cna.gov.co/1741/articles-186370_ley_3092.pdf

Congreso de Colombia (1996). Decreto 111. Por el cual se compilan la Ley 38 de 1989, la Ley 179 de 1994 y la Ley 225 de 1995 que conforman el Estatuto Orgánico del Presupuesto. Disponible en: http://www. secretariasenado.gov.co/senado/basedoc/decreto_0111_1996.html

Economía Planeta. 1980. Diccionario Enciclopédico. Barcelona: Planeta

Gitman, Lawrence (2000). Principios de Administración Financiera. México: Editorial Pretince-Hill.

Urrego Gleidy y Gutierrez Jahir (2018). Hacia la gestión y saneamiento financiero y fiscal de los municipios del departamento de Antioquia. Tend. vol.19 Disponible en: http://www.scielo.org.co/scielo. php?script=sci_arttext \&pid=S0124-86932018000200113\&lang=es

Ministerio de Educación Nacional (2018). Sistema Nacional de Información de la Educación Superior. Colombia Ramírez, Alejandro (1998): Hacienda Pública. Colombia: Editorial Temis, S.A.

Restrepo, J. C. (2000). Hacienda pública. Bogotá: Universidad Externado de Colombia.

Sánchez, A. (2006). Gestión Financiera. Disponible en: http://www.eumed.net/tesis-doctorales/2006/ asc/3h.htm

Sistema Universitario Estatal del Caribe Colombiano - SUE (2019). Instituciones de Educación Superior. Disponible en: https://suecaribe.edu.co/sue-caribe/universidades/

Philippatos, G. (974). Fundamentos de Administración Financiera, México: Ed. Mc Graw Hill.

Van Horne, James. (1988). Fundamentos de Administración Financiera. México: Editorial Pretince-Hill.

Villanueva, A., Rico, F., y Rico, H. (2015). Economía pública. Recuperado en: http://bonga.unisimon.edu.co/ handle/20.500.12442/1210. Colombia 